\title{
Design of Chaotic Orthogonal Discrete Frequency Coding Waveform
}

\author{
Baisen Liu' ${ }^{1,2, a}$ and Wulin Zhang ${ }^{3, b}$
}

${ }^{1}$ College of Electrical and Information Engineering, Heilongjiang Institute of Technology, Harbin, China

${ }^{2}$ School of Electronics and Information Engineering, Harbin Institute of Technology, Harbin, China

${ }^{3}$ College of Information and Communication Engineering, Harbin Engineering University, Harbin, China

aSped_liu@126.com, bwl19820212@live.cn

Keywords: MIMO; Discrete frequency encoding; Chaotic; Auto-correlation; Cross-correlation

\begin{abstract}
The orthogonality of the transmitting signal is crucial for implementing multiple input multiple output (MIMO) radar system. The chaotic orthogonal discrete frequency coding waveform (DFCW) can be utilized to MIMO radar, and generate a large amount of orthogonal signal with random lengths and quantities. This paper applies the continuous chaotic system to analyze autocorrelation and crosscorrelation for the DFCW design of MIMO radar. The analysis indicates the side lobe peak of autocorrelation is 0.21 , and the peak of crosscorrelation decreases with increasing the code length in DFCW without repetition of coding coefficients. The DFCW correlations are similar in continuous chaotic system and discrete chaotic system. In DFCW with repetition of coding coefficients, the side lobe peak of autocorrelation and the peak of crosscorrelation decrease as the code length is increased. The correlation of continuous chaotic system with coding coefficients repetition, is better than the correlation of discrete chaotic system, so it is the relatively ideal transmitting waveform for MIMO radar.
\end{abstract}

\section{混沌正交离散频率编码波形的设计

\author{
刘柏森 $^{1,2, a}$, 张雾琳 $^{3, b}$ \\ 1. 黑龙江工程学院电气与信息工程学院, 哈尔滨 150000 \\ 2. 哈尔滨工业大学电子与信息工程学院，哈尔滨 150000 \\ 3. 哈尔滨工程大学信息与通信工程学院, 哈尔滨 150000 \\ a sped_liu@126.com, bw119820212@live.cn
}

摘要: 发射信号的正交性好坏是多输入多输出 (MIMO) 雷达实现的关键, 混沌正交离散频率 编码波形 (DFCW) 是 MIMO 雷达可以利用的正交信号, 并且可以大量产生任意长度和数量的 正交信号。本文将连续混沌系统引入 MIMO 雷达正交 DFCW 的设计, 对其自相关及互相关性 进行了分析。分析表明编码系数不重复 DFCW 的的自相关旁瓣峰值为 0.21 , 其互相关峰值随 码长的增大而降低, 并且基于连续混沌和离散混沌的 DFCW 的相关性近似; 编码系数重复 DFCW 的自相关旁瓣峰值和互相关峰值随码长的增大而降低, 基于连续混沌系统的编码系数重复 DFCW 的相关性较好，优于离散混沌系统，是较为理想的 MIMO 雷达发射波形。

关键词：多输入多输出；离散频率编码关键词；混沌；自相关；互相关

\section{1. 引言}

MIMO 雷达作为一种新兴的、先进的有源目标检测技术，其目标检测性能和低截获能力优于传 统雷达，这些优势都得益于空间分集和波形分集 [1] [2]。当各天线发射波形理想正交时，MIM0 雷达可有效形成虚拟孔径，从而提高了雷达的目标检测性能 [3]。发射机发射的波形是相互正 
交的，可以克服多个发射波形间相互串扰的问题。因此，发射信号的正交性是 MIMO 雷达实现 的关键技术。

正交频分复用信号、正交相位编码信号 [4] [5]、正交离散频率编码信号 (DFCW) [6]等正交波 形已经取得了一定的研究成果, 但上述正交波形设计方法存在以下问题: 随着发射端发射阵 元的增多及正交波形的数量的增加, 波形的正交性随之下降; 如果信号编码的长度较长, 则 导致优化算法的计算量巨大，耗时长、效率低、求解也相当复杂。

混沌信号的非周期类随机性，使得基于混沌的雷达波形具有近似 $\delta$ 函数的自相关函数和近似 为 0 的互相关函数, 因此在正交波形设计中具有天然的优势, 近年来引起广泛关注并得到较 多研究。目前, 基于混沌的正交波形设计大多采用离散混沌映射进行信号调制 [7]- [10], 但 是离散混沌系统参数少, 初值的取值范围小, 产生机制简单, 设计自由度小, 并且离散混沌 系统状态变量的概率分布一般为近似均匀分布和 arc-sin 分布, 而连续混沌系统状态变量的 概率分布一般为近似高斯分布和凹状分布, 因此将连续混沌时间序列应用于正交波形是否能 够获得更好的性能需要进行研究。本文将连续混沌系统应用于正交离散频率编码波形的设计, 对基于连续混沌系统调制所产生的 DFCW 的自相关性和互相关性进行了研究, 分析了基于连续 混沌的 DFCW 的相关性与编码长度之间的关系。

\section{2. 离散频率编码波形数学模型}

离散频率编码波形 (DFCW) 包含个子脉冲, 每个子脉冲采用不同的频率编码, 通过对频率编码 的合理设计, 可以得到很低的互相关峰值, 使 DFCW 波形间相互正交, 这种波形称为正交 DFCW 波形, 由 $m$ 个 DFCW 波形组成的正交波形集的数学表达为

$$
\begin{gathered}
\left\{s_{m}(t)=\sum_{n=0}^{N-1} p_{n}^{m}(t-n T)\right\}, m=1,2, \cdots, M \\
p_{n}^{m}(t)= \begin{cases}\exp \left(j 2 \pi f_{n}^{m} t\right), & 0 \leq t \leq T \\
0, & t \geq T\end{cases}
\end{gathered}
$$

其中, $\mathrm{T}$ 是子脉冲时间宽度, $\mathrm{N}$ 是频率编码序列长度, 即子脉冲数, $f_{n}^{m}=c_{n}^{m} \Delta f$ 为波形集第 $m$ 个 波形中第 $n$ 个子脉冲的频率编码, $c_{n}^{m}$ 为编码系数, 表示频率编码的顺序, $c_{n}^{m} \in\{0,1, \cdots, N-1\}$, $\Delta f=1 / T$ 。

基于混沌序列的正交离散频率编码波形, 通过不同初值生成 $M$ 组混沌序列, 时间序列的状态 变量为 $x \in[a, b]$, 对序列进行量化, 量化级为子脉冲数。根据量化后时间序列取值所在的区间, 得到相应的编码系数。量化方式不同, 生成的正交 DFCW 分为两种。当生成时间序列的状态变 量按从小到大排列, 一一对应编码系数, 由于混沌具有非周期性和伪随机性, 混沌序列中每 一点值都不相同, 因此使得对应的编码系数点各不相同, 没有重复的编码系数, 即为编码系 数不重复的正交 DFCW。当量化方式为均匀量化时, 量化后时间序列的状态变量出现重复, 所 在的区间可能落入多个编码系数, 也可能没有落入编码系数, 即编码系数重复的正交 DFCW。

\section{3. 离散频率编码波形相关性能分析}

\section{1 . 自相关函数}

设正交 DFCW 的自相关函数为:

$$
R_{m m}(\tau)=\frac{1}{N T} \int_{-\infty}^{\infty} s_{m}^{*}(t) s_{m}(t-\tau) d t
$$

其中, $\tau$ 为延时。将式 (1) 代入式 (3) 得到: 


$$
R_{m m}(\tau)=\frac{1}{N T} \int_{-\infty}^{\infty} \sum_{n=0}^{N-1} p_{n}^{m *}(t-n T) p_{n}^{m}(t-\tau-n T) t+\frac{1}{N T} \int_{-\infty}^{\infty} \sum_{n=0}^{N-1} \sum_{h=0, h \neq n}^{N-1} p_{n}^{m *}(t-n T) p_{h}^{m}(t-\tau-h T) d t
$$

设波形 $s_{m}(t)$ 中 $p_{n}^{m}(t)$ 与 $p_{h}^{m}(t)$ 的互相关函数为:

$$
\phi_{n h}(\tau)=\frac{1}{T} \int_{-\infty}^{\infty} p_{n}^{m *}(t) p_{h}^{m}(t-\tau) d t
$$

将式(2) 代入式 (5) 得到:

$$
\phi_{n h}^{m}(\tau)= \begin{cases}\frac{T-|\tau|}{T} \frac{\sin \pi \alpha(T-|\tau|)}{\pi \alpha(T-|\tau|)} \exp \left(-j \pi \alpha(T-\tau)-j 2 \pi f_{n} \tau\right),|\tau \leq T| \\ 0, \quad|\tau>T|\end{cases}
$$

其中 $\alpha=f_{n}^{m}-f_{h}^{m}$ 。当 $n=h$ 时, 式 (6) 为 $p_{n}^{m}(t)$ 的自相关函数, 如式 (7) 所示:

$$
\phi_{n n}^{m}(\tau)=\left\{\begin{array}{l}
\frac{T-|\tau|}{T} \exp \left(-j 2 \pi f_{n} \tau\right),|\tau \leq T| \\
0, \quad|\tau>T|
\end{array}\right.
$$

将式(6)、式(7)代入式(4) 得到:

$$
R_{m m}(\tau)=\left\{\begin{array}{lc}
\frac{1}{N} \sum_{n=0}^{N-1}\left(\phi_{n n}(\tau)+\sum_{h=0, h \neq n}^{N-1} \phi_{n h}(\tau-(n-h) T)\right) \quad|\tau| \leq T \\
0, & |\tau|>T
\end{array}\right.
$$

DFCW 的自相关函数由子脉冲的自相关函数和互相关函数决定，其中互相关函数部分为:

$$
\begin{aligned}
\frac{1}{N} \sum_{n=0}^{N-1} \sum_{h=0, h \neq n}^{N-1} \phi_{n h}(\tau-(n-h) T)= & \frac{T-|\tau|}{N T} \sum_{n=0}^{N-1} \sum_{h=0, h \neq n}^{N-1} \frac{\sin \pi\left(f_{n}^{m}-f_{h}^{m}\right)(T-|\tau|)}{\pi\left(f_{n}^{m}-f_{h}^{m}\right)(T-|\tau|)} \\
& \cdot \exp \left(-j \pi\left(f_{n}^{m}-f_{h}^{m}\right)(T-\tau)-j \pi f_{h}^{m} \tau\right)
\end{aligned}
$$

当 $\tau \rightarrow 0$ 时, 上式近似等于:

$$
\begin{aligned}
\frac{1}{N} \sum_{n=0}^{N-1} \sum_{h=0, h \neq n}^{N-1} \phi_{n h}(\tau-(n-h) T, 0) & \approx \frac{1}{N} \sum_{n=0}^{N-1} \sum_{h=0, h \neq n}^{N-1} \frac{\sin \pi\left(c_{n}^{m}-c_{h}^{m}\right) \Delta f T}{\pi\left(c_{n}^{m}-c_{h}^{m}\right) \Delta f T} \exp \left(-j \pi\left(c_{n}^{m}-c_{h}^{m}\right) \Delta f T\right) \\
& =\frac{1}{N} \sum_{n=0}^{N-1} \sum_{h=0, h \neq n}^{N-1 \neq n} \frac{\sin \pi \Delta c \Delta f T}{\pi \Delta c \Delta f T} \exp (-j \pi \Delta c \Delta f T)=0
\end{aligned}
$$

其中, $\Delta c$ 为不为零的整数。因此, 式 (9) 可以改写为:

$$
R_{n m}(\tau)=\left\{\begin{array}{c}
\frac{T-|\tau|}{N T}\left(\sum_{n=0}^{N-1} \frac{\sin \pi N \tau / T}{N \sin \pi \tau / T} \exp \left(-j \pi(N-1) \frac{\tau}{T}\right)+\left(\sum_{a \in A}^{N-1} \exp \left(-j 2 \pi f_{a}^{m} \tau\right)-\sum_{b \in B}^{N-1} \exp \left(-j 2 \pi f_{b}^{m} \tau\right)\right)\right)|\tau| \leq T \\
0, \\
|\tau|>T
\end{array}\right.
$$

其中 $A$ 为重复频率编码的集合, $B$ 为编码频率没有被使用的编码集合。显然, 当 $\tau=0$ 时自相 关函数出现峰值, 如果没有 ${ }^{T-|\tau|}$ 项, 在 $\tau=T$ 时也将出现峰值。当 $N$ 值较大时,

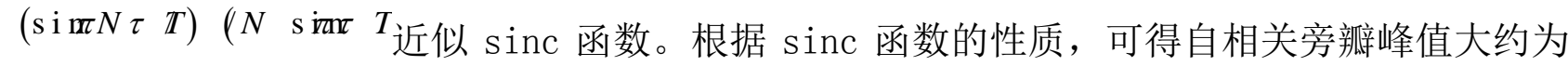
0.21 。因此, 编码系数不重复的 DFCW 的自相关旁瓣几乎与频率编码的顺序无关, 仅与码长有 微小的关系。因此, 编码系数重复的正交 DFCW 的自相关旁瓣峰值可以表示为: 


$$
\max \left(0.21+\frac{T-|\tau|}{N T}\left(\sum_{a \in A}^{N-1} \exp \left(-j 2 \pi f_{a}^{m} \tau\right)-\sum_{b \in B}^{N-1} \exp \left(-j 2 \pi f_{b}^{m} \tau\right)\right)\right)
$$

由式 (12) 可以知道, 当频率编码序列满足特定排列时, 编码系数重复的正交 DFCW 的自相关旁 瓣峰值可以小于 0.21 , 因此, 编码系数重复的 DFCW 的自相关旁瓣与频率编码的顺序紧密相关, 通过对编码系数重复的正交 DFCW 的设计, 可以获得更低的自相关旁瓣峰值。

3. 2. 互相关性

设 $s_{p}(t)$ 和 $s_{q}(t)$ 为 DFCW 正交波形集中的任意两个波形，则两个波形的互相关函数为:

$$
\begin{aligned}
R_{p q}(\tau)= & \frac{1}{N T} \int_{-\infty}^{\infty} \sum_{n=0}^{N-1} p_{n}^{p *}(t-n T) \sum_{n=0}^{N-1} p_{n}^{q}(t-\tau-n T) d t \\
= & \frac{1}{N T} \int_{-\infty}^{\infty} \sum_{n=0}^{N-1} \exp \left(j 2 \pi f_{n}^{q}(t-\tau-n T)-j 2 \pi f_{n}^{p}(t-n T)\right) \\
& +\frac{1}{N T} \int_{-\infty}^{\infty} \sum_{n=0}^{N-1} \sum_{h=0, h \neq n}^{N-1} \exp \left(j 2 \pi f_{h}^{q}(t-\tau-h T)-j 2 \pi f_{n}^{p}(t-n T)\right) d t
\end{aligned}
$$

因为频率编码信号是基于连续混沌时间序列生成的, 因为混沌具有遍历性和伪随机性, 当 $N \rightarrow \infty$ 时, 有:

$$
\begin{aligned}
& \lim _{N \rightarrow \infty} \int_{-\infty}^{\infty} \sum_{n=0}^{N-1} \exp \left(j 2 \pi f_{n}^{q}(t-\tau-n T)-j 2 \pi f_{n}^{p}(t-n T)\right) d t=0 \\
& \lim _{N \rightarrow \infty} \int_{-\infty}^{\infty} \sum_{n=0}^{N-1} \sum_{h=0, h \neq n}^{N-1} \exp \left(j 2 \pi f_{h}^{q}(t-\tau-h T)-j 2 \pi f_{n}^{p}(t-n T)\right) d t=0
\end{aligned}
$$

则当 $N \rightarrow \infty$ 时, 式 (13) 趋近于 0。因此, 编码系数不重复 DFCW 正交波形具有准正交性。从式 (13) 可以得知, 互相关函数与频率编码序列密切相关, 而频率编码序列由连续混沌时间序列 量化得到, 因此为了得到信号间良好的准正交性, 需要考虑不同连续混沌系统生成的时间序 列对频率编码序列的影响, 从而选择准正交性能最好的连续混沌系统。虽然连续混沌时间序 列都具有伪随机性，但不同连续混沌系统，具有不同的分布规律和统计特性，因此基于不同 连续混沌时间序列设计的编码系数不重复 DFCW 正交雷达波形性能也可能是不同的。

\section{4. 数值仿真与结果分析}

假设编码系数不重复 DFCW 正交雷达波形, 编码长度为 $N$, 子脉冲时间宽度为 $100^{\mu s}$, 离散频 率间隔 $\Delta f=0.01 \mathrm{MHz}$, 采样率为 $2 \Delta f \square N$ 。 
表 4.1 自相关旁瓣峰值随码长的变化特性

\begin{tabular}{llllll}
\hline $\begin{array}{l}\text { 混 沌 序 } \\
\text { 列 }\end{array}$ & & 64 & 128 & 256 & 512 \\
\hline Lorenz & 0.1999 & 0.2058 & 0.2090 & 0.2106 & 0.2114 \\
Rossler & 0.2000 & 0.2058 & 0.2089 & 0.2106 & 0.2114 \\
Chua & 0.1996 & 0.2058 & 0.2089 & 0.2106 & 0.2114 \\
Liu & 0.2000 & 0.2059 & 0.2089 & 0.2106 & 0.2114 \\
Bernoul & 0.1997 & 0.2058 & 0.2090 & 0.2106 & 0.2114 \\
li & & & & & \\
Logisti & 0.1999 & 0.2059 & 0.2089 & 0.2106 & 0.2114 \\
c & & & & & \\
Tent & 0.1999 & 0.2058 & 0.2089 & 0.2106 & 0.2114 \\
\hline
\end{tabular}

表 4.2 互峰值随码长的变化特性相关

\begin{tabular}{llllll}
\hline 混沌序列 & 32 & 64 & 128 & 256 & 512 \\
\hline Lorenz & 0.1067 & 0.0618 & 0.0328 & 0.0185 & 0.0098 \\
Rossler & 0.1183 & 0.0684 & 0.0376 & 0.0198 & 0.0119 \\
Chua & 0.1327 & 0.0714 & 0.0416 & 0.0232 & 0.0128 \\
Liu & 0.1093 & 0.0635 & 0.0332 & 0.0181 & 0.0094 \\
Bernoulli & 0.1064 & 0.0624 & 0.0322 & 0.0183 & 0.0096 \\
Logistic & 0.1443 & 0.0766 & 0.0438 & 0.0267 & 0.0144 \\
Tent & 0.1117 & 0.0619 & 0.0350 & 0.0184 & 0.0098 \\
\hline
\end{tabular}

表 4. 1、表 4.2 分别为基于连续混沌序列和离散混沌序列的编码系数不重复 DFCW 的自相关旁 瓣峰值的平均值和互相关峰值的平均值 (100 次平均) 随混沌编码长度 $\mathrm{N}$ 的变化, 连续混沌时 间序列的时间间隔为 $10-3 \mathrm{~s}$, 选取状态变量 $x$ 的时间序列。从表 4.1、表 4.2 可以得出随着码 长 $\mathrm{N}$ 的增大, 自相关旁瓣峰值基本没有变化, 因此自相关旁瓣峰值与频率编码没有关系, 只 与码长有微弱的关系, 这与前面的分析相一致。互相关峰值随码长 $\mathrm{N}$ 的增大而减小, 基于各 混沌系统的编码系数不重复 DFCW 的互相关性能比较相近。

表 3、表 4 分别为基于连续混沌时间序列和离散混沌序列的编码系数重复 DFCW 的自相关旁瓣 峰值的平均值和互相关峰值的平均值 (100 次平均) 随混沌编码长度 $\mathrm{N}$ 的变化, 其中连续混沌 时间序列的时间间隔为 10-3s。从表 3 可以得出随着码长 $\mathrm{N}$ 的增大, 自相关旁瓣峰值逐渐降 低, 自相关旁瓣峰在不同码长条件下有较大差异, 因此自相关旁瓣峰值与频率编码是密切相 关的，这与前面的理论分析相一致。其中基于 Lorenz 系统, Liu 系统和 Rossler 系统的 DFCW 的自相关旁瓣峰值随码长 $\mathrm{N}$ 的增大而减小, 当码长大于 256 时, 自相关旁瓣峰值低于 0.21 , 自相关性优于基于离散混沌系统的编码系数重复 DFCW。由表 4 可知, 编码系数重复 DFCW 具 有很好的准正交性。其中基于连续混沌系统的编码系数重复 DFCW 的互相关性能比较接近, 准 正交性能优于基于离散混沌系统的编码系数重复 DFCW。其中, 基于 Lorenz 系统和 Liu 系统 的编码系数重复 DFCW 具有更好的自相关性能和互相关性能, 可以作为一种正交波形应用。 
表 3 自相关旁瓣峰值随码长的变化特性

\begin{tabular}{llllll}
\hline 混沌序列 & 32 & 64 & 128 & 256 & 512 \\
\hline Lorenz & 0.3122 & 0.2152 & 0.2108 & 0.1725 & 0.1358 \\
Rossler & 0.3317 & 0.2225 & 0.2144 & 0.1850 & 0.1546 \\
Chua & 0.5700 & 0.5996 & 0.6027 & 0.6052 & 0.6053 \\
Liu & 0.3185 & 0.2113 & 0.2110 & 0.1653 & 0.1338 \\
Bernoulli & 0.3025 & 0.2478 & 0.2142 & 0.2100 & 0.2095 \\
Logistic & 0.3698 & 0.3048 & 0.2706 & 0.2634 & 0.2574 \\
Tent & 0.3206 & 0.2851 & 0.2200 & 0.2141 & 0.2136 \\
\hline
\end{tabular}

表 4 互相关峰值随码长的变化特性

\begin{tabular}{llllll}
\hline 混沌序列 & 32 & 64 & 128 & 256 & 512 \\
\hline Lorenz & 0.1100 & 0.0647 & 0.0355 & 0.0193 & 0.0106 \\
Rossler & 0.1491 & 0.0867 & 0.0517 & 0.0259 & 0.0134 \\
Chua & 0.1246 & 0.0711 & 0.0410 & 0.0227 & 0.0121 \\
Liu & 0.1144 & 0.0664 & 0.0361 & 0.0211 & 0.0111 \\
Bernoulli & 0.1769 & 0.1068 & 0.0648 & 0.0363 & 0.0222 \\
Logistic & 0.2026 & 0.1354 & 0.0772 & 0.0471 & 0.0144 \\
Tent & 0.1762 & 0.1128 & 0.0666 & 0.0392 & 0.0217 \\
\hline
\end{tabular}

\section{5. 结论}

本文将连续混沌系统引入 MIMO 雷达正交 DFCW 的设计, 对其自相关及互相关性进行了分析, 并分析了基于连续混沌的 DFCW 的相关性与编码长度之间的关系。分析表明编码系数不重复 DFCW 的的自相关旁瓣峰值与混沌序列的排序无关, 约为 0.21 , 其互相关峰值随码长的增大而 降低, 并且基于连续混沌和离散混沌的 DFCW 的自相关和互相关性能近似; 编码系数重复 DFCW 的自相关旁瓣峰值和互相关峰值随码长的增大而降低, 基于连续混沌 Lorenz 系统, Rossler 系统和 Liu 系统的编码系数重复 DFCW 的相关性较好, 优于基于离散混沌系统的编码系数重复 DFCW，是较为理想的 MIMO 雷达发射波形。

\section{6. 致谢}

本文由基金项目：黑龙江省自然科学基金（F201322）支持。

\section{参考文献}

[1] Eran Fishier, Alex Haimovich, Rick Blum, et a1.. MIMO radar: whose time has come[C].Proceeding of the IEEE Radar Conference, 2004, 71-78.

[2] 明文华, 刘志学. 一种新体制雷达-MIMO雷达[J]. 火控雷达技术, 2008, 37(1):10-13.

[3] 何子述, 韩春林, 刘波. MIMO雷达概念及其技术特点分析 [J]. 电子学报, 2005，33(12): 2441-2445

[4] Deng H. Polyphase code design for orthogonal netted radar systems[J]. IEEE Trans. on Signal Processing, 2004, 52(11): 3125-3135.

[5] Liu B., He Z., Zeng J., et al.. Polyphase orthogonal code design for MIMO radar systems[C].International Conference on Radar, 2006, 1-4. 
[6] 刘波, 何子述, 王海江. MIMO雷达中的DFCW 设计及性能分析 [J]. 电子科技大学学报, 39(5), September, 2010: 688-691.

[7] 杨进, 邱兆坤, 黎湘, 庄钊文. 混沌随机步进频率信号分析与处理[J]. 国防科技大学学报, 2012, 34(6): 163-169.

[8] 蒋飞, 刘中, 胡文, 包伯成. 任意频谱结构的连续混沌调频雷达波形设计 [J]. 电子学报, 2010, 09(38): 2195-2198.

[9] 丁凯，杨汝良. 混沌调频雷达信号仿真[J]. 电子与信息学报, 2006, 28(2):354-357.

[10]林云生, 武文, 王晓军, 等. 一类混沌二相编码脉冲压缩的性能分析 [J]. 系统工程与电子 技术, 2003, 25(4): 489-491.

\section{Acknowledgements}

This work is supported by Natural Science Foundation of Heilongjiang Province (No. F201322).

\section{References}

[1] Eran Fishier, Alex Haimovich, Rick Blum, et a1.. MIMO radar: whose time has come[C].Proceeding of the IEEE Radar Conference, 2004, 71-78.

[2] Chen H., Li X., Zhuang z.. A Rising Radar System MIMO Radar [J]. ACTA ELECTRONICA SINICA, 2012, 40(6):1190-1196.

[3] He Z., Han C., Liu B.. MIMO Radar and Its Technical Characteristic Analyses [J]. ACTA ELECTRONICA SINICA, 2005,33 (12): 2441-2445.

[4] Deng H.. Polyphase code design for orthogonal netted radar systems [J]. IEEE Trans. on Signal Processing, 2004, 52(11): 3125-3135.

[5] Liu B., He Z., Zeng J., et al.. Polyphase orthogonal code design for MIMO radar systems[C].International Conference on Radar, 2006, 1-4.

[6] Liu B., He Z., Wang H..DFCW Signal Design and Performance Analysis for MIMO Radar [J]. Journal of University of Electronic Science and Technology of China, 2010, 39 (5): 688-691.

[7] Yang J., Qiu Z., et al.. Analysis and processing of the chaotic-based random stepped frequency signal [J]. Journal of National University of Defense Technology, 2012, 34(6): 163-169.

[8] JIANG F., LIU Z., et al.. Waveform Design of Arbitrary Spectrum Structure for Continuous-Chaos Frequency-Modulating Radar [J]. ACTA ELECTRONICA SINICA, 2010, 09(38): 2195-2198.

[9] Ding K., Yang R.. Chaotic FM Radar Imaging Simulation [J]. JOURNAL OF ELECTRONICS \& INFORMATION TECHNOLOGY, 2006, 28(2):354-357.

[10]Lin Y., Wu W., et al.. Analysis of Pulse Compression Performance for a Type of Chaotic Biphase Codes [J]. SYSTEMS ENGINEERING AND ELECTRONICS, 2003, 25(4): 489-491.

作者简介：刘柏森(1979-), 男, 黑龙江省哈尔滨市, 副教授, 信号与信息处理, sped_liu@126.coml。 